\title{
The Phase Diagram of Iron and the Temperature of the Inner Core
}

\author{
Orson L. ANDERSON \\ Center for Earth and Planetary Interiors, Institute of Geophysics and Planetary Physics, \\ UCLA, Los Angeles, CA 90024-1567, U.S.A.
}

(Received December 14, 1992; Revised March 25, 1993; Accepted April 6, 1993)

Birch was the pioneer of understanding the earth's core: that the core was mostly iron; that the solid inner core was nearly pure iron; that the liquid core had most of the impurities; and that the phase diagram (p.d.) of pure iron was complicated with several phases and triple points (t.p.). In 1972 he said, " $5000^{\circ} \mathrm{K}$, for the central temperature ... may have some standing as an upper limit." I reconstruct the phase diagram accounting for new experiments and theories arising in the subsequent two decades.

I stretch Birch's p.d. to account for the experiments of BOEHLER et al. (1990), BRown and MCQUEEN (B\&M) $(1982,1986)$, and Yoo et al. $(1992,1993)$. BoEHLER $(1993)$ reports the $\epsilon$ - $\gamma$-liquid t.p. at $100 \mathrm{GPa}$. This t.p. plus the solid-solid (ss) transition at $200 \mathrm{GPa}$ reported by B\&M require an additional high $T$ phase, $\alpha^{\prime}$, which has been suggested to be bcc. The new $\alpha^{\prime}$ phase requires another t.p. near $190 \mathrm{GPa}$, which implies that the inner core is made largely of bcc iron. I suggest a $T_{m}$ of about $6500^{\circ} \mathrm{K}$ for pure iron at the inner core-outer core boundary pressure. The $T_{m}$ of the inner core itself will be affected by possible inner core impurities and by outer core impurities of larger concentration. To paraphrase Birch, $5700^{\circ} \mathrm{K}$ for the central temperature may have some standing as an upper limit.

\section{Introduction (The $\epsilon$ - $\gamma$-Liquid Triple Point)}

Debate about the phase diagram of iron has centered on location of the triple point joining the $\epsilon$ (hcp), $\gamma$ (fcc), and liquid phase. As a quick review of the history of this $\gamma-\epsilon-\ell$ (gammaepsilon-liquid) triple point, seven solutions are presented in Fig. 1.

BIRCH's (1972) extrapolation located it at $100 \mathrm{GPa}$ and $3575^{\circ} \mathrm{K}$. LIU's (1975) extrapolation placed the triple point at $92.5 \mathrm{GPa}$ and $3240^{\circ} \mathrm{K}$. ANDERSON's (1986) extrapolation placed it at $280 \mathrm{GPa}$ and $5480^{\circ} \mathrm{K}$. That same year BoEHLER's (1986) extrapolation placed it at $70 \mathrm{GPa}$ and $2700^{\circ} \mathrm{K}$. Williams et al.'s (1991) extrapolation placed it at $300 \mathrm{GPa}$ and $7700^{\circ} \mathrm{K}$. BOEHLER et al.'s (1990) work and BoEHLER's later (1992) work located the triple point at "about" 100 $\mathrm{GPa}$ and $2800^{\circ} \mathrm{K}$. BOEHLER's most recent experimental finding (1993) locates the triple point at exactly $100 \mathrm{GPa}$ and $2900^{\circ} \mathrm{K}$.

BIRCH (1972) first attempted to find this triple point; his careful discussion of the physics and thermodynamics of the phase diagram makes his paper seminal. He made the following assumptions: for the iron fcc phase, $(\gamma): d K / d P=6, \alpha K_{T}$ independent of pressure, $K_{T}=90$ $\mathrm{GPa}, \alpha=70 \times 10^{-6} / \mathrm{K}$ at $1970^{\circ}$ (at the beginning of the $\gamma$ phase), and he took from then current high-pressure measurements the value $(d T / d P)_{m}=42^{\circ} \mathrm{K} / \mathrm{GPa}$. In terms of measurable quantities, Birch's equation for $(\partial T / \partial V)_{m}$ is

$$
\left(\frac{\partial T}{\partial V}\right)_{m}=-\left(\frac{V_{0}}{V}\right)\left[\frac{K_{T}\left(\frac{\partial T}{\partial P}\right)_{m}}{V}\right]\left[1-\alpha K_{T}\left(\frac{\partial T}{\partial P}\right)_{m}\right],
$$

where the subscript $m$ is for melting; $K_{T}$ is the isothermal bulk modulus; and $\alpha$ is the volume thermal expansivity. Using (1), Birch converted the measured value of $(\partial T / \partial P)_{m}$ to $(\partial T / \partial V)_{m}$ so that he could plot his curve and make his extrapolations in the $T-V$ plane. Birch's work shows 
firm understanding of the thermodynamics involved, because this kind of plot clarifies so much of the physics. One advantage of the $T-V$ plot is that the $(\partial T / \partial V)$ data lie nearly on a straight line, making extrapolation easy. Using reports of the experimental values, he evaluated (1). Knowing the slope in the $T-V$ plane at low pressure, he calculated the melting curve according to the then popular KRAUT and KENNEDY (1966) melting law, which is given as

$$
\Delta T_{m}=T_{0}\left[1+\left(\frac{1}{V_{0}}\right)\left(\frac{d T}{d V}\right) \Delta V\right] .
$$

Thus Birch's melting "curve" was a straight line in $T-V$ space.

BIRCH's (1972) phase diagram in $V-T$ space is shown in Fig. 2. The stippled zone is the $\Delta V$ of the phase transitions. The $T-V$ plot shows $\Delta V$ across the phase transitions and illustrates the change of $\Delta V$ with $T$ and $V$ at phase boundaries, all of which is hidden in the $T-P$ plot. The slope of the $T-V$ line showing the $\gamma$-liquid boundary is simply the straight line representing the KrautKennedy law with Birch's evaluated constants. The decrease of $\Delta V$ at higher $T$ along the melting curve is calculated by obtaining $d T_{m} / d P$ and the calculated $\Delta S$ from the Lindemann law using the Clausius-Clapeyron equation. $\Delta S$ along the liquidus may be calculated from the randomness of the arrangement of atoms in a liquid using statistical principles. If the Hugoniot data permit, a value of $\Delta V$ may be found experimentally at the transitions to constrain the calculation. The $T-V$ diagram illustrates an important point well-known in physical thermodynamics: the sum of the $\Delta V$ 's around the triple point must equal zero, so $\Delta V$ on the high side of the triple point equals the sum of the solid-liquid (s- $\ell) \Delta V$ plus the $\Delta V$ of the solid-solid transition below. The sum of $\Delta S$ around the triple point must also be zero. This procedure is of great use in calculating $d T_{m} / d P$ on the high side of a triple point.

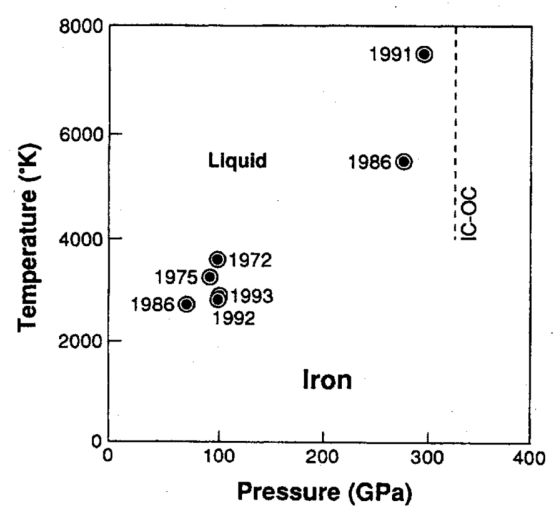

Fig. 1.

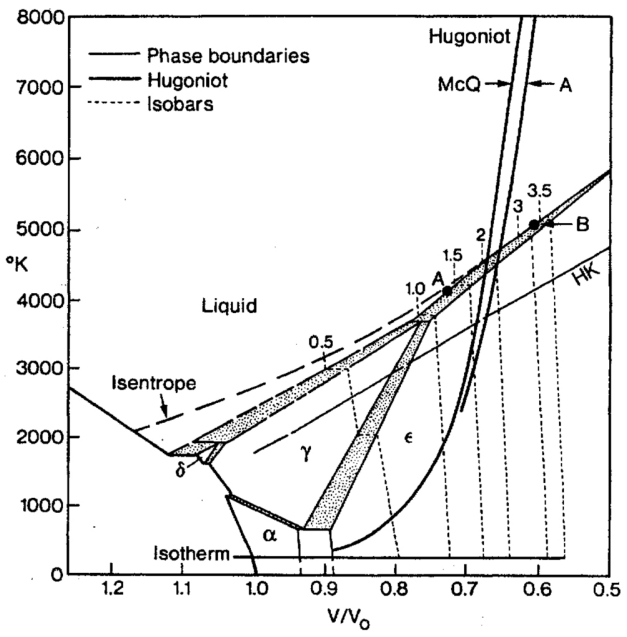

Fig. 2.

Fig. 1. Historical representation of the location of the liquid- $\epsilon$ (hcp) $-\gamma$ (fcc) triple point: BIRCH (1972); LIU (1975); BoeHler (1986), lower; ANderson (1986), upper; Williams et al. (1991); Boehler (1992, 1993). The BOEHLER (1993) triple point value is a measurement; all others are extrapolations of lower pressure data.

Fig. 2. The BIRch (1972) phase of iron shown in $T-V$ space. A represents the core-mantle boundary pressure, and $\mathrm{B}$ represents the inner-outer core pressure. The Hugoniots are labeled after the shock wave work of McQueen and Altshuler. Stippled areas represent the $\Delta V$ of transition between the various phases. Under this interpretation, the central core is $\epsilon$ (hcp) iron (after BIRCH, 1972). 
LIU (1975) reevaluated the position of the triple point. By this time some new results on the melting of iron in the $\gamma-\ell$ phase boundary were available as a result of a study by LIU and BASSETT (1975). These authors also remeasured the slope of the $\gamma$-liquid boundary and found a different value from that used by Birch. LIU (1975) then followed Birch's procedure, using the Kraut-Kennedy law for $T$ versus $V$; but with differing constants, and extrapolated to find his value for the triple point, which was not too far from Birch's pressure or temperature (see Fig. 1).

Following Birch's lead in calculating results for presentation on a $T-V$ plane, ANDERSON (1986) reevaluated the position of the $\gamma-\epsilon-\ell$ triple point. He found it to be much higher in both pressure $(280 \mathrm{GPa})$ and temperature $\left(5760^{\circ} \mathrm{K}\right)$ using (incorrectly) shock compression results that had been presented in the previous decade. He also used the Lindemann law of melting, which allows one to interpolate between two points in the $T-P$ plane, so that if the slope $d T / d P$ at the initial point and the value of $\gamma$ at the initial point are known, $d T / d P$ can be found all along the curve, including the upper point. This technique allows us to find $d T / d P$ at the high triple point for the liquidus of the upper branch (the $(\epsilon-\ell)$ branch in Fig. 2) using

$$
\left(\frac{d \ln T_{m}}{d P}\right)=\left(\frac{2}{K_{T}}\right)\left(\gamma-\frac{1}{3}\right)
$$

In the early 1980's the Lindemann law had been in dispute. STEvenson (1981) and StACEY and IRVINE (1977) derived a slightly modified form of the Lindemann law, the former using liquid state theory and the latter using thermodynamic equilibrium equations based on the ClausiusClapeyron equation. The corrections they found to Lindemann's law were inconsequential and justify the use of the original form of the Lindemann law. POIRIER (1986) rederived Lindemann's law with minor adjustments using dislocation theory and applied it specifically to iron, finding $T_{m}$ at the IC-OC boundary pressure to be $6000 \pm 300^{\circ} \mathrm{K}$ (see also PoIRIER and SHANkLAND (1993)).

Using Lindemann's law of melting and the relationship between the Grüneisen parameter $(\gamma)$ and density $(\rho)$ as $\gamma / \gamma_{0}=\left(\rho / \rho_{0}\right)^{q}$, where $q$ is a constant, the melting equation relating $T_{m}$

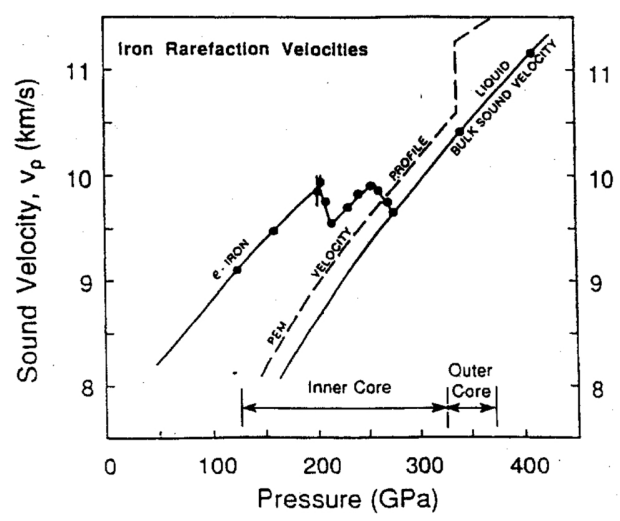

Fig. 3. The sound velocity $\left(v_{P}\right)$ versus $P$ experiments on iron of BRown and MCQUEEN $(1982$, 1986), which demonstrated two transitions at high pressure. For the transition at $243 \mathrm{GPa}, v_{P}$ drops and becomes equal to the bulk sound velocity. This indicates that the shear velocity is zero at pressures higher than $243 \mathrm{GPa}$. Thus this transition indicates a boundary between a solid and a liquid phase. For the transition at $200 \mathrm{GPa}$, $v_{S}$ also drops, but not to zero, so this is interpreted as marking a solid-solid phase transition. (After BRown and MCQUEen, 1986.) 
and $\rho$ was found to be (ANDERSON, 1986)

$$
\frac{T_{m}}{T_{m 0}}=\left(\frac{\rho}{\rho_{0}}\right)^{2 / 3} \exp \left\{\left(\frac{2 \gamma_{0}}{q}\right)\left[1-\left(\frac{\rho_{0}}{\rho}\right)^{q}\right]\right\} .
$$

Further, BRown and MCQUEen $(1982,1986)$ found a solid-solid transition in the Hugoniot of iron at $200 \mathrm{GPa}$, which they interpreted as the $\epsilon-\gamma$ transition (see Fig. 3). Following Brown and McQueen's idea that the $\epsilon-\gamma$ transition passes through $200 \mathrm{GPa}$, ANDERSON (1986) located the $\epsilon-\gamma-\ell$ triple point slightly below $200 \mathrm{GPa}$ (see Fig. 1). We now know that the $\epsilon-\gamma$ transition occurs at much lower pressure. An important contribution of the Brown and McQueen experiment was the location of a solid-liquid transition at $243 \mathrm{GPa}$.

BOEHLER (1986) presented results on the triple point derived from his own measurements on a laser heated sample in a diamond anvil cell. He found that the slope, $d T_{m} / d P$, of the $\gamma-\ell$ boundary decreased with $P$ in the $T$ - $P$ plane, but his values confirm BundY's (1965) $d T_{m} / d P$ for the $\gamma-\epsilon$ line $\left(28^{\circ} \mathrm{K} / \mathrm{GPa}\right)$ at low pressures. BOEHLER's 1986 extrapolated value for the triple point based on experiments to $43 \mathrm{GPa}$ was at $70 \mathrm{GPa}$ and $2700^{\circ} \mathrm{K}$. BoEHLER's (1992) results based on experiments to $110 \mathrm{GPa}$ showed experimentally that there appears to be an $\epsilon-\gamma-\ell$ triple point, at "about" $100 \mathrm{GPa}$ and $2800^{\circ} \mathrm{K}$. His 1993 result based on experiments to $200 \mathrm{GPa}$ definitely showed that the $\epsilon$ - $\gamma$-liquid triple point is located at $100 \mathrm{GPa}$ and $2900^{\circ} \mathrm{K}$ (BOEHLER, 1993).

\section{The Phase Diagram to $200 \mathrm{GPa}$ : The Diamond Anvil Cell Experiments}

Boehler's statically determined melting curve on the $\gamma$-liquid branch of Fe has been confirmed by high pressure measurements using the diamond anvil cell (DAC) by the Uppsala group, SHEN et al. (1993), see Fig. 4. The DAC work on $T_{m}$ of iron by the Livermore shock wave group has appeared in two places: Yoo et al. $(1992,1993)$ (see Fig. 4). Figure 4 also shows the comparison between the Berkeley group (Williams et al., 1987) DAC measurement of $T_{m}$ versus $P$ and those of BoEHLER et al. (1990), the Uppsala group (1993), and the Livermore group (1992, 1993). The Canberra group, RINGwOod and HiBBERSON (1990), reported the $T_{m}$ of iron at $16 \mathrm{GPa}$ to be $2218 \mathrm{~K}$ using a multi-anvil apparatus, finding very good agreement with BoEHLER et al. (1990).

Figure 4 shows that researchers from four independent laboratories agree upon the statically determined values of $T_{m}$ in the $\gamma$-liquid boundary, and that their results depart sharply from the Williams et al. result at about $40 \mathrm{GPa}$. A choice has to be made in the construction of the phase diagram. Because of the weight of experimental evidence, I choose to disregard the Berkeley group data in the construction of the phase diagram. For an evaluation of the experimental method of measuring $T_{m}$ by BOEHLER et al. (1990) and BOEHLER (1992) versus those of the Berkeley group (1987), see DubA (1992).

The low pressure part of the phase diagram-below $160 \mathrm{GPa}$ - which involves static compression including the diamond anvil cell, the piston-cylinder apparatus, and the large volume press is shown in Fig. 5. The solution of the $\epsilon$ - $\gamma$-liquid phase diagram shown in Fig. 5b satisfies the thermodynamic constraints of a well behaved triple point. This shows that the Boehler data, as well as those by Yoo et al. and Shen et al., join up almost perfectly with the low pressure data and the low pressure phase diagram (Fig. 5a) solutions published more than a decade ago (Bundy, 1965; MAO et al., 1967; STRONG et al., 1973; LiU, 1975). Boehler's measured value of the $\epsilon-\gamma-\ell$ triple point in Fig. 5b is placed near that of the older determination of Birch (Fig. 1), thus indicating the serendipity of BIRCH (1972), who worked from a much more limited database.

3. The New High Temperature Phase, $\alpha^{\prime}$

Since the $\epsilon$ - $\gamma$-liquid triple point is at $100 \mathrm{GPa}$, the $\gamma$ phase is restricted to pressures less than $100 \mathrm{GPa}$. How then can we explain the solid-solid transition seen by Brown and McQueen at 


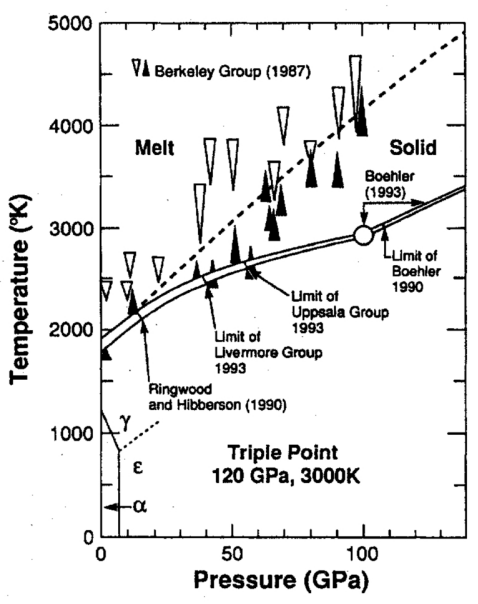

Fig. 4.

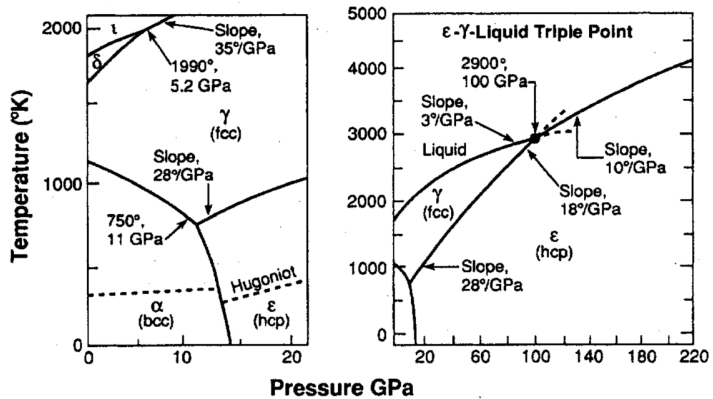

Fig. 5 .

Fig. 4. The statically measured melting curves of iron from BoEHLER et al. (1990), BOEHLER (1993), SHEN et al. (1993), called the Uppsala group; and Yoo et al. (1992), called the Livermore group, plotted on the graph presented by WiLliams et al. (1987), called the Berkeley group. The Boehler, Uppsala group and the Livermore group data points confirm each other and depart from the Berkeley group data points at about $50 \mathrm{GPa}$. The melting curve line suggested by the Berkeley group (solid curve) departs from the others at about $20 \mathrm{GPa}$. The cross shows the RINGwOOD and HIBBERSON (1990) melting point determined using their multi-anvil press. The liquid $-\epsilon-\gamma$ triple point is defined by BOEHLER (1993).

Fig. 5. The pressure-temperature phase diagram of iron showing values for $P<20 \mathrm{GPa}$ (a); and the recent location of the $\epsilon$ - $\gamma$-liquid triple point, (b). In (a), the $\delta-\ell-\gamma$ triple point (at $1990^{\circ} \mathrm{K}$ and $5.2 \mathrm{GPa}$ ) is from MAO et al. (1967); the $\gamma$-liquid slope is from LIU (1975); the $\epsilon$ - $\gamma$ slope at the triple point is from BuNDY (1965); the location of the $\alpha-\epsilon-\gamma$ triple point (at $11 \mathrm{GPa}$ and $750^{\circ} \mathrm{K}$ ) is from STRONG et al. (1973). In (b) the location of the $\gamma-\epsilon-\ell$ triple point $\left(100 \mathrm{GPa}\right.$ and $\left.2900^{\circ} \mathrm{K}\right)$ is from BOEHLER (1993); the path of the $\gamma$-liquid boundary is from SHen et al. (1993), Yoo et al. (1992, 1993), BoEHLER et al. (1990), and Boehler (1993). The path of the $\epsilon$-liquid boundary is from Boenlen (1993); the slope of the $\gamma-\epsilon$ boundary at the triple point is by the author. Note that the $\epsilon$-liquid line just above the triple point is between the projections of the $\epsilon-\gamma$ boundary and the $\gamma$-liquid boundary, thus satisfying thermodynamic constraints on the location of the triple point.

$200 \mathrm{GPa}$, since all of the standard phases except $\epsilon$ are below $100 \mathrm{GPa}$ ? What is the other phase that forms a boundary with $\epsilon$ above about $200 \mathrm{GPa}$ ? As early as 1986, Boehler remarked that the B\&M transition at $200 \mathrm{GPa}$ is "not the $\epsilon-\gamma$ transition, but another new $\epsilon-\Theta$ transition," thus bringing the possibility of a new high-pressure phase into the phase diagram of iron. BOEHLER (1986) said, "a search for an additional iron phase transition at room temperature near 2 Mbar is suggested."

Ross et al. (1990) used theoretical arguments that lend credence to the existence of a fifth high-pressure phase. Their arguments were based upon the plausibility, for transition metals, of a stable bcc solid phase at high temperatures that forms a boundary with the melting line. This idea has received much attention since it was introduced by AlEXANDER and MCTAGUE (1978). Young and Grover (1984), using a simple exponential law for atomic repulsion in the calculation of the compression of iron, came to the conclusion that a bcc structure is stable at high temperature. Ross et al. (1990) presented a great deal of circumstantial evidence to show that the boundary of this new bcc phase $\left(\alpha^{\prime}\right)$ with the $\epsilon$ phase is nearly parallel with the $\mathrm{P}$ axis, and they assumed that it could be moved up to intersect with the solid-solid transition observed by Brown and McQueen at $200 \mathrm{GPa}$.

The theory of MATSUI (1992) supports this new high $T$ bcc phase, $\alpha^{\prime}$. Using molecular 
dynamics methods, Matsui found a first order phase change from hcp to bcc at $5000^{\circ} \mathrm{K}$ for 300 $\mathrm{GPa}$, as shown in Fig. 6 . The $\Delta V / V$ he found was $0.5 \%$, the volume of the bcc phase being lower (see Fig. 7).

The experimental and theoretical data above $200 \mathrm{GPa}$ (with reported error bars) that are used for construction of the upper part of the phase diagram are assembled in Fig. 7. The experimental data are based on shock wave results, except for one datum by BOEHLER (1993) at $200 \mathrm{GPa}$. The lower $P$ data of Boehler are shown as continuous lines, for reference.

The shock compression results of Yoo et al. (1993) are shown in Fig. 7. We note in particular that the lower limit of the error bars of the Yoo et al. result comes close to the upper limit of the error bars of the B\&M result at $243 \mathrm{GPa}$. The shock compression data for iron in the very high pressure region reported by BAss et al. (1990) and AHREns et al. (1990) are also shown in Fig. 7.

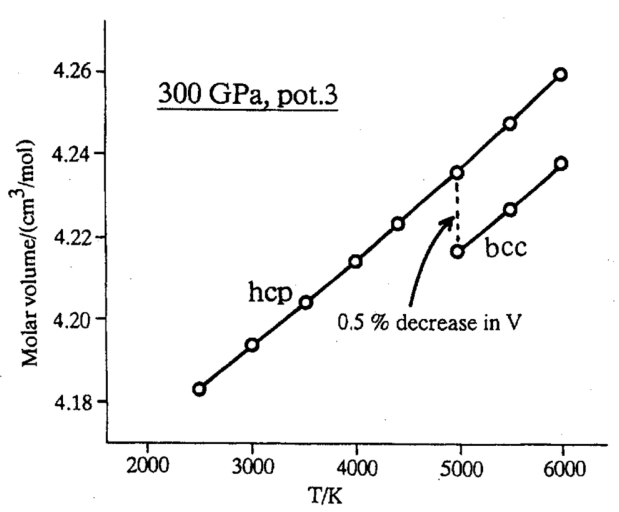

Fig. 6.

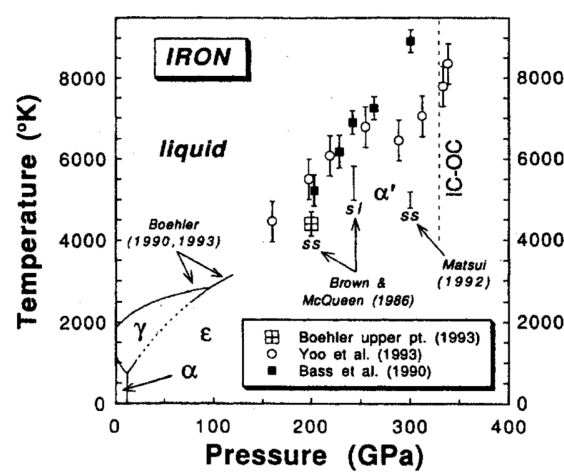

Fig. 7.

Fig. 6. The transition from hcp iron to bcc iron at high pressure (300 GPa) found by simulation studies of MATSUI (1992) using molecular dynamics methods. This is a first order transition with a value of $\Delta V / V$ of $0.5 \%$ The volume of the bcc phase is less than that of the hcp phase (after MATSUI, 1992).

Fig. 7. The selected experimental and theoretical data for $P>150 \mathrm{GPa}$, which together define the phase diagram of iron at extreme pressure. ss stands for a solid-solid transition and s $\ell$ stands for a solid-liquid transition. The empty circles and full squares indicate values of $T_{m}$ as found from Livermore and CalTech shock compression studies. The two Brown and McQueen points represent values found from the Los Alamos shock compression group (see Fig.3). There is agreement between the two Livermore and CalTech shock compression data for $P<250 \mathrm{GPa}$, but not above that pressure. Also shown is the uppermost static measurement of BOEHLER (1993) at $200 \pm 20 \mathrm{GPa}$, giving $T_{m}=4200 \pm 200^{\circ} \mathrm{K}$.

\section{Construction of the Upper Part of the Phase Diagram of Pure Iron}

All the data in Fig. 7 are consistent with placing a triple point $\left(\epsilon-\alpha^{\prime}\right.$-liquid) in the region of $190 \mathrm{GPa}$ near $4100^{\circ} \mathrm{K}$, much like the suggestion of Ross et al. (1990). The exact location of this triple point is tentative because the high point datum from Boehler's new DAC experiment lies at the same pressure reported by Brown and McQueen for the solid-solid transition. Nevertheless there is a solution for the triple point that satisfies the error bars of the B\&M solid-solid transition at $200 \mathrm{GPa}$ and those of the Boehler solid-liquid transition at $220 \mathrm{GPa}$. This is shown in the insert of Fig. 8. We use the upper error bar limit for these BOEHLER (1993) data and the lower error bar limit for the Brown and McQueen data. 


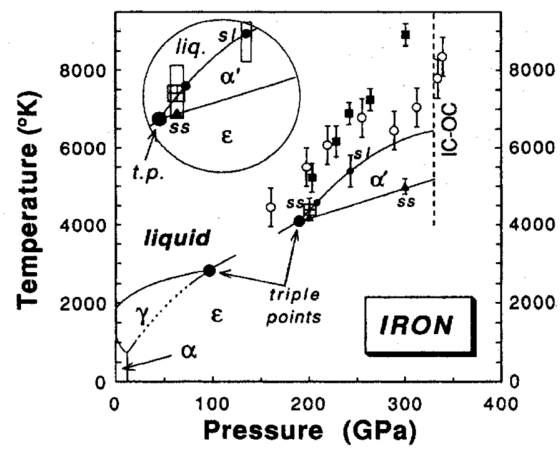

Fig. 8. The phase diagram of iron in T-P space that best satisfies the data set in Fig. 7. $\alpha^{\prime}$ (hcp) is the solid phase at inner core conditions defined by the $\alpha^{\prime}-\epsilon$-liquid triple point at $190 \mathrm{GPa}$ and $4100^{\circ} \mathrm{K}$. The insert shows the details of the branches of the triple point near their juncture. The $\alpha^{\prime}$-liquid boundary is found by the Lindemann law, so chosen that the boundary passes within the limits of the s- $\ell$ transition at $243 \mathrm{GPa}$ (BOEHLER, 1993) and the lower limit of the Brown and McQueen datum at $243 \mathrm{GPa}$ and passes just below the lower limit of the Yoo et al. (1993) data. The liquidus of pure iron is $6500 \pm 300^{\circ} \mathrm{K}$ at the inner core-outer core boundary. The solid triangles define the solid-solid boundary of the $\epsilon$ and $\alpha^{\prime}$ phases.

A choice must be made between the various sets of shock wave data to find the $\alpha^{\prime}$-liquid boundary. The location of the Boehler datum at $220 \mathrm{GPa}$ and $4400^{\circ} \mathrm{K}$ requires one to choose either the $\mathrm{B} \& \mathrm{M} \mathrm{s}-\ell$ point or the shock wave data lying above $6500^{\circ} \mathrm{K}$ for the path of the liquidus. I take the position that the trajectory of $T_{m}(P)$ passing from the high triple point ( $\epsilon$ - $\alpha^{\prime}$-liquid) to the IC-OC boundary pressure must pass through the limits given by the B\&M solid-liquid transition at $243 \mathrm{GPa}$ and close to the lower limits of the Yoo et al. shock compression result near the IC-OC boundary pressure, as seen in Fig. 8. Then, using Eqs. (3) and (4), a curve representing the Lindemann melting law is passed through the midpoint of the 243 GPa Brown and McQueen solid-liquid error bars, finding an intercept at the inner core-outer core boundary between the limits of $6800^{\circ} \mathrm{K}$ and $6200^{\circ} \mathrm{K}$, giving $6500 \pm 300^{\circ} \mathrm{K}$. This is shown in Fig. 8 .

\section{A Pure Iron Inner Core}

The adiabatic compression in the inner core results in an increase in $T$ of about $200^{\circ}$ from the IC-OC boundary pressure to the earth central pressure. In a core made of $\alpha^{\prime}$ iron, the impurity concentration should be small, even though a core made of $\epsilon$ iron would require much more impurity (ANDERSON, 1986). Nevertheless, the impurities of an $\alpha^{\prime}$ central core, even though small in concentration, would result in a freezing point depression. I assume that the adiabatic compression increase of $T_{m}$ will nearly cancel the freezing point depression effect of $T_{m}$ for the $\alpha^{\prime}$ case. For an examination of effects of impurities in an $\epsilon$-iron core on the density of the inner core, see JEPHCOAT and OLSEN (1987).

In a solid-liquid boundary with significant impurities in the liquid phase, the effective melting temperature of the solid phase is controlled by the temperature of the liquid phase, where there will definitely be a freezing point depression. According to POIRIER and SHANkLand (1993), the melting temperature of the inner core at the ICB will be the $T_{m}$ of pure iron less the freezing point depression due to about $10 \%$ light impurities, e.g., a drop of about 500 to $1000^{\circ} \mathrm{K}$. Choosing a mid value of their proposed freezing point depression, I recommend a $T_{m}$ of the inner core (including the earth's center) of about $5700^{\circ} \mathrm{K}$.

It is instructive to look at the $V-T$ version of the phase diagram because we can identify parameters involved in the thermodynamics of heat flow (see Fig. 9). Note that the $\gamma-\ell$ and the 
$\alpha^{\prime}-\ell$ boundaries plot as a straight line in $T-V$ space. We note that the $\Delta V$ of the liquid- $\epsilon$ boundary is larger than found in either of the lower branches, as the result of the condition $\sum \Delta V=0$ around the triple point. We can explain the high slope of $d T_{m} / d P$ in the upper branch of the $\epsilon-\gamma-\ell$ t.p. by the Clausius-Clapeyron equation

$$
\frac{d T_{m}}{d P}=\frac{\Delta V}{\Delta S}
$$

In the upper branch $\Delta V$ is larger than in the lower solid-liquid branch. If $\Delta S$ does not change significantly, then the larger $\Delta V$ will lead to a higher $d T_{m} / d P$ in the upper branch, as seen in Fig. 8.

Further, according to Matsui (Fig. 6), in the highest t.p., the volume decreases at the $\epsilon$ - $\alpha^{\prime}$ branch going from $\epsilon$ to $\alpha^{\prime}$, having the opposite sign to that of the $\Delta V$ in the $\epsilon$-liquid branch, but it is a small effect. This volume decrease, though small, requires that the $\Delta V$ of the liquid branch be the sum of $\Delta V$ of the $\epsilon-\alpha^{\prime}$ branch and the $\epsilon-\ell$ branch, and thus slightly larger than the $\Delta V$ of the $\epsilon-\ell$ branch. Thus the $\Delta V$ for the $\alpha^{\prime}$-liquid branch is slightly larger than the $\Delta V$ of the $\epsilon$-liquid branch, at least near the triple point. This suggests that the slope of $d T_{m} / d P$ above the

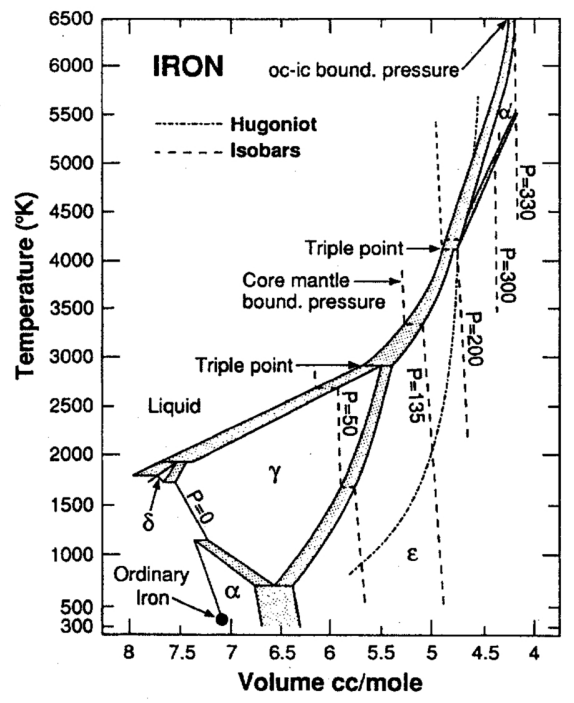

Fig. 9.

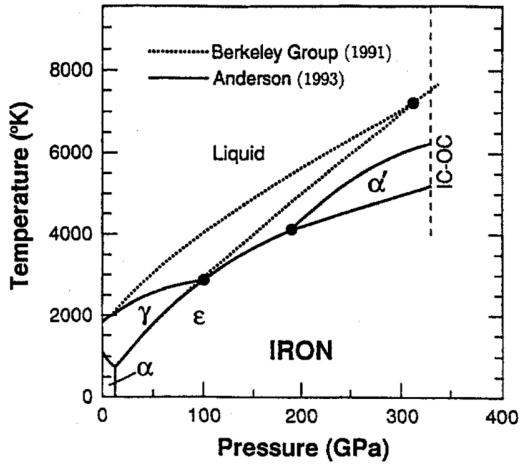

Fig. 10 .

Fig. 9. The phase diagram of iron in $T-V$ space, constructed from Fig. 8. Notable features include: 1) the large $\Delta V$ of the top branch of the $\epsilon-\gamma$-liquid triple point, which accounts for the large value of $d T_{m} / d P$ for this branch; 2) the relatively large $\Delta V$ of the top branch of the $\alpha^{\prime}-\epsilon$-liquid triple point, which suggests a large heat of crystallization at the OC-IC boundary pressure; and 3) straightness of the $\gamma$-liquid boundary, suggesting that the $\Delta V$ and $\Delta S$ increase at the same rate along the boundary. The suggested $\epsilon$ - $\alpha^{\prime}$-liquid triple point is $190 \mathrm{GPa}$ and $4100^{\circ} \mathrm{K}$ (after ANDERSON, 1990).

Fig. 10. A comparison of the Berkeley group (WILliams et al., 1991) phase diagram of iron (dotted line) and the phase diagram of iron proposed here (solid line) (ANDERson, 1992). The essential differences are: 1) the Williams et al. diagram has 4 phases and 3 triple points (two are compressed on the $T$ axis, but see Fig. $5 \mathrm{a}$ ), while the proposed phase diagram has 5 phases and 4 triple points. The 5 th phase, existing at the highest solid pressure, is proposed to be $\alpha^{\prime}(\mathrm{bcc})$. In the Williams et al. diagram $\epsilon$ (hcp) iron comprises the inner core; while in the proposed diagram, the fifth phase (perhaps $\alpha^{\prime}(\mathrm{bcc})$ ) comprises the inner core. In the dotted phase diagram, $7700^{\circ} \mathrm{K}$ is the value of $T_{m}$ at the IC-OC boundary $P$, in contrast to $6500^{\circ} \mathrm{K}$ for the proposed phase diagram. The Anderson reference is to this paper. 
$\epsilon$ - $\alpha^{\prime}$-liquid triple point is only slightly larger than the slope $d T_{m} / d P$ just below the $\epsilon$ - $\alpha^{\prime}$-liquid triple point, as seen in Fig. 8. The value of $\Delta V$ at the IC-OC boundary shown in Fig. 8 is slightly less than found by ANDERSON (1986)) for a different phase diagram of iron. If they are the same, the value of $\Delta H$, the heat of crystallization at the solid-liquid iron boundary is 200 to $370 \mathrm{cal} / \mathrm{gm}$ (ANDERson and Young, 1988). This is a rather high value of $\Delta H$. Taking the lower limit of the Anderson and Young solution for $\Delta H$, we find the lower limit of their calculation for heat production for this phase diagram. That is $4 \times 10^{12}$ Watts for heat production from crystallization of the inner core (ANDERSON and YOUNG, 1988).

The liquid-solid boundary of iron in $T$-P space consists of two highly curved arcs and one arc with slight curvature. Is this unusual? The liquid boundaries found in the phase diagram of sulfur, for example, are very complicated (YounG, 1991), suggesting that the structure of the proposed phase diagram of iron as shown in Figs. 8 and 9 is not to be considered as out of the question.

In Fig. 10 we compare the phase diagram of iron proposed by WiLLIAMS et al. (1991) with that proposed here. In the Williams et al. version: 1) $\epsilon$ is the inner core phase; and 2) the temperature of pure iron at the IC-OC boundary pressure is $7700^{\circ} \mathrm{K}$ rather than $6500^{\circ} \mathrm{K}$ as proposed here. An $\epsilon$ core at inner core conditions will have a density larger (by about $0.6 \mathrm{gm} / \mathrm{cc}$ ) than the PREM density of the inner core (see Fig. 2 of ANDERSON, 1982). A similar result was found by JEPHCOAT and OLSEN (1987). Thus the $\epsilon$ phase requires a larger concentration of impurities in the inner core, whereas the density of the $\alpha^{\prime}$ phase for the inner core will probably agree with the density of PREM for the inner core. Thus the proposed solution for the phase diagram will probably allow a virtually pure iron or iron with a small amount of nickel to comprise the inner core.

\section{The Question of Impurities in the Outer Core}

What we now know about the phase diagram of pure iron (and it is considerably more than a decade ago) will not necessarily help us when we need to construct the phase diagram of an alloy of iron such as $\mathrm{Fe}-\mathrm{FeO}$ and including nickel.

From experiments of BOEHLER (1992) it appears that below about $40 \mathrm{GPa}$ the $T_{m}$ of FeO is roughly equal to the $T_{m}$ of iron. However, triple points will complicate determination of the $T_{m}-P$ properties of an FeO-Fe mixture at conditions of the deep core. An alloy of iron will almost certainly have a shifted position of the triple points from that of pure iron, and perhaps no triple points at all. To extrapolate the $T_{m}$ of $\mathrm{FeO}$ in order to understand the Fe-FeO alloy while totally ignoring any possible high $P$ triple points could be hazardous.

All of the experiments and theories that have contributed to understanding the phase diagram of pure iron will have to be performed again for $\mathrm{FeO}, \mathrm{FeS}$ and $\mathrm{FeH}$ in the 100-300 GPa range. A fundamental theory of binary solutions will have to be developed in order to understand how to choose between eutectics and solid solutions in order to find $T_{m}$ at arbitrary composition and $P$. This binary theory must be extended to tertiary solutions and perhaps even quaternary solutions to handle an outer core with arbitrary amounts of the solutes $\mathrm{FeS}, \mathrm{FeO}, \mathrm{FeH}$, carbon, and nickel added to pure iron.

We can speculate on what the effects of impurities of $\mathrm{FeO}, \mathrm{FeS}$, and $\mathrm{NiO}$ do to the value of $T_{m}$ of pure iron at very high pressure. The experiments of BOEHLER (1992) up to $50 \mathrm{GPa}$ tell us that FeS lowers $T_{m}$ when mixed with Fe, but that FeO raises $T_{m}$ when mixed with Fe (at least above $20 \mathrm{GPa}$ ). There is the intriguing possibility that the effect of the presence of $\mathrm{FeO}$ may counteract to some extent the effect that $\mathrm{FeS}$ and $\mathrm{FeH}$ have on the depression of $T_{m}$ in outer core conditions. 
Table 1. Predicted temperatures of the melting point of pure iron at the pressure of the inner-outer core boundary.

\begin{tabular}{|c|c|}
\hline & $T_{m}\left({ }^{\circ} \mathrm{K}\right)$ \\
\hline GILVARRY (1957) & 6200 \\
\hline ZHARKOV (1962) & 6200 \\
\hline BUNDY and STRONG (1962)* & $6100-8100$ \\
\hline HIGGENS and KENNEDY $(1971)^{*}$ & 4250 \\
\hline BIRCH $(1972)^{*}$ & 5100 \\
\hline LEPPALUOTO (1972) & $7000-9000$ \\
\hline BoschI (1975) & 6000 \\
\hline $\operatorname{LIU}(1975)$ & 5125 \\
\hline STACEY (1977) & 3157 \\
\hline BoscHI et al. (1979) & $4500-7000$ \\
\hline ABELSON $(1981) \ddagger$ & 7800 \\
\hline STEVENSON (1981) & 6300 \\
\hline BROWN and MCQUEEN (1982)§ & $6200 \pm 500$ \\
\hline ANDERSON $(1982) \S$ & $5900 \pm 700$ \\
\hline SPILIOPOULOS and STACEY $(1984) \S$ & $6140 \pm 575$ \\
\hline YounG and Grover (1984) & 6600 \\
\hline ANDERSON $(1986) \S$ & $6210 \pm 400$ \\
\hline POIRIER (1986) & $6000 \pm 300$ \\
\hline BROWN and MCQUEEN (1986)§ & $5800 \pm 500$ \\
\hline Ross et al. (1990) & 6400 \\
\hline WILLIAMS et al. (1987) & 7700 \\
\hline POIRIER and SHANKLAND (1993) & $\begin{array}{l}6160 \pm 250(\epsilon) \\
5610 \pm 240\left(\alpha^{\prime}\right)\end{array}$ \\
\hline This report $\S$ & $6500 \pm 300$ \\
\hline
\end{tabular}

*Based on an extrapolation from experiments at $6 \mathrm{GPa}$.

$\ddagger$ Based upon the Monte Carlo theory.

$\S$ Based upon an extrapolation from experiments at $243 \mathrm{GPa}$.

\section{The Temperature of Iron at the Outer Core-Inner Core Boundary}

Examination of Fig. 8 suggests that as a working hypothesis $T_{m}$ of pure iron at IC-OC boundary conditions is near $6500^{\circ} \mathrm{K}$. Below is a list of values of $T_{m}$ at $330 \mathrm{GPa}$ for pure iron as predicted by various workers. Most of the early estimates were based upon melting of a single phase, without consideration of the complexity of the actual phase diagram. We note that extrapolations to the IC-OC boundary constrained by the Brown and McQueen solid-liquid boundary ( $243 \mathrm{GPa})$ marked by $\S$ yield quite similar values of $T_{m}$. It would appear that the B\&M solid-solid boundary places strong constraints on the presence of the 5th phase of iron, and that their solid-liquid boundary (200 GPa) places strong constraints on the melting temperature of pure iron at $330 \mathrm{GPa}$. The focus of the arguments on $T_{m}$ for the high pressure region then shifts to the certainty of the temperature on the $B \& M$ s- $\ell$ boundaries used in this report.

\section{8. $T_{m}$ for the Brown and McQueen Solid-Liquid Transition at $243 \mathrm{GPa}$}

The pressures measured by Brown and McQueen are quite secure. But could the temperatures they report for the $243 \mathrm{GPa}$ transition, a window we use for extrapolation to the 330 pressure limit, be insecure? The basic problem in estimating temperatures in a shock compression analysis concerns the calculation of temperature found from thermodynamics from the Hugoniot. The 
thermodynamic evaluation of the change in $T$ along the Hugoniot is found from

$$
d T=-\frac{\gamma T}{V} d V+\frac{\left(V_{0}-V\right) d P+\left(P-P_{0}\right) d V}{2 C_{V}},
$$

where the first term is a correction along an isentrope from an increment in $V$, and the second represents the shift from the isentrope back to the Hugoniot resulting from that increment (MCQueEn, 1964).

We review the method of calculating the temperature along the isentrope centered at $V_{0}$. To find the isentropic correction, the isentropic version of $\gamma$, the Grüneisen ratio, is used

$$
\gamma=-\left(\frac{\partial \ln T}{\partial \ln V}\right)_{S},
$$

which yields the temperature at $V$ along an isentrope, by

$$
T_{S}=T_{0} \exp \left\{-\int_{V_{0}}^{V} \gamma \frac{d V}{V}\right\} .
$$

To evaluate (7) one needs to know how $\gamma / V$ changes with $V$ at high $P$, high $T$. This is often found by using the power law relationship

$$
\frac{\gamma}{\gamma_{0}}=\left(\frac{V_{0}}{V}\right)^{q}
$$

where $\gamma_{0}$ and $\rho_{0}$ represent ambient conditions. It is conventional wisdom in shock wave analysis to assume that $q=1$, so that $\gamma$ at $P$ and $T$ is found from $\gamma\left(0, T_{0}\right)$, the measured gamma at $P=0$, and at some reference temperature, $T_{0}$. For $q=1,(8)$ becomes

$$
\gamma(P, T) / V(P, T)=\gamma\left(0, T_{0}\right) / V\left(0, T_{0}\right)
$$

The high pressure value of $\gamma(P, T)$ determined by assuming the low pressure value of $q$ (such as $q=0.6$ for iron measured by BOEHLER and RAMAKRISHNAN (1980)) is not warranted. ANDERSON et al. (1993) showed by a thermodynamic theory that $q$ should always be near 1 at high $P$ and $T$, supporting the conventional wisdom of shock wave data reduction methods. However, the value of $\gamma_{0}$ for $\epsilon$ iron is not well known. BRown and MCQUEEN (1986) took limits of $\gamma_{0}$ between 1.7 and 2.0 and found little change in the resulting $T_{m}$. Therefore the question of the uncertainty of $\gamma_{0}=\gamma\left(0, T_{0}\right)$ may slightly bias the B\&M result on $T_{m}$, although the limits on $\gamma_{0}$ appear reasonable.

To evaluate (5) at high $P$ and $T, C_{V}$ must be determined. In an insulator, in the quasiharmonic approximation, $C_{V}$ is independent of $P$ and $T$ at extreme conditions, but for iron there is the problem of determining the electronic contribution to $C_{V}$. This correction has been calculated by researchers from several laboratories including JAMIESON et al. (1978). BROWN and MCQUEEN (1986) examined the magnitude of $C_{V}$ including the electronic correction term for iron in detail, and plausible bounds for $C_{V}$ were found. The error bars in $T_{m}$ resulting from uncertainties in $\gamma_{0}, q$, and $C_{V}$ were set at $\pm 500^{\circ} \mathrm{K}$. The B\&M result for $T_{m}$ for the s- $\ell$ transition is $5800^{\circ} \mathrm{K}\left( \pm 500^{\circ} \mathrm{K}\right)$, and as seen in Fig. 8 is lower than the other shock wave results. It is difficult to dismiss the Brown and McQueen results for $T_{m}$ in favor of the other shock wave results for $T_{m}$ because the other shock experiments, while directly measuring radiance due to melting, also suffer because the thermal diffusivity of the window next to the iron specimen through which the radiance must pass is unmeasured at high $P$ and $T$. That is to say, in deducing $T_{m}$ from the measured radiance, detailed computations involving the thermal diffusivity effect have to be made. 
(See discussion of this point in MCQUEEN and IsAAK, 1990.) Thus there are problems in data reduction of all the shock wave methods (TAN and AHRENS, 1990), because each method has to rely on detailed and complex calculations in the data reduction in which unmeasured quantities must be estimated. Thus a choice must be made (see Section 4 ) between the various shock wave data points in choosing the path of the $\alpha^{\prime}$-liquid boundary.

\section{Summary}

All of the data presented, excepting the static DAC work of the Berkeley group (1987), form a coherent pattern from which a phase diagram of iron is constructed. To warp the phase diagram so as to include the Berkeley group data (and the projection of $T_{m}$ versus $P$ resulting therefrom) would require one to disregard the works of BROWN and MCQUEEN (1986), BOEHLER et al. (1990), Ross et al. (1990), Boehler (1992, 1993), Matsui (1992), Shen et al. (1993), Yoo et al. (1992), and RINGWOOD and HiBBERSON (1990). Disregarding the Berkeley group (1987) data on $T_{m}$ in favor of other DAC results leads to the establishment of the $\epsilon$ - $\gamma$-liquid triple point at $100 \mathrm{GPa}$ and $2900^{\circ} \mathrm{K}$.

The experiments and theories at very high pressure (above $200 \mathrm{GPa}$ ) are each very difficult and represent the present state of the art. Consequently each of them is subject to some individual criticism because they involve some uncertainty in the computations arising in the data reduction. But taken collectively, as shown in Fig. 8, they tend to reinforce each other and create a fabric that is hard to deny. My choice for the $\alpha^{\prime}$-liquid boundary that best satisfies this collection of shock wave data leads to a value of $6500^{\circ} \mathrm{K}$ for the $T_{m}$ of pure iron at a pressure of the IC-OC boundary of the core. Nevertheless, new experiments may someday be presented that will warp the phase diagram sufficiently to change the value of $T_{m}$ at the IC-OC pressure, so $6500^{\circ} \mathrm{K}$ must be considered as tentative. Using the value of $6500^{\circ} \mathrm{K}$ for pure bcc iron, it is estimated that the temperature of the inner core, including the earth's center, is near $5700^{\circ} \mathrm{K}$, the lowering of $T_{m}$ arising from freezing point depression in the liquid core at the IC-OC boundary.

I thank R. Boehler, C.-S. Yoo, and S. Saxena for providing me with abstracts, preprints, and information in correspondence in advance of their own publications. I thank T. Yagi, D. Young, D. G. Isaak, and J. P. Poirier for helpful comments on an early version of the typescript. Financial support was provided by NSF through grant EAR-91-17280. IGPP paper no. 3903.

\section{REFERENCES}

Abelson, R. S., Monte Carlo calculations of the melting point of iron at core pressures, Ph.D. Thesis, University of California at Los Angeles, 1981.

Ahrens, T. J., H. TAN, and J. D. BAss, Analysis of shock temperature data for iron, High Pressure Res., 2, 145-157, 1990.

Alexander, S. and J. MCTAGUe, Should all crystals be bcc? Landau theory of solidification and crystal nucleation, Phys. Rev. Lett., 41, 702-705, 1978.

Anderson, O. L., The earth's core and phase diagram of iron, Philos. Trans. R. Soc. London, Ser. A, 306, 21-35, 1982.

Anderson, O. L., Properties of iron at the earth's core conditions, Geophys. J. R. Astron. Soc., 84, 5461-5579, 1986.

Anderson, O. L., The high-pressure triple points of iron and their effects on the heat flow from the earth's core, J. Geophys. Res., 95, 21697-21797, 1990.

Anderson, O. L., Francis Birch and the phase diagram of iron (Abstract), Eos Trans. AGU, 73, 67-68, 1992.

ANDERson; O. L. and D. A. Young, Crystallization of the earth's inner core, in Structure and Dynamics of Earth's Deep Interior, Geophys. Monogr. Ser., Vol. 39, edited by D. E. Smylie and R. Hides, pp. 83-90, AGU, Washington, DC, 1988.

Anderson, O. L., H. OdA, A. Chopelas, and D. IsAaK, A thermodynamic theory of the Grüneisen ratio at extreme conditions: $\mathrm{MgO}$ as an example, Phys. Chem. Minerals, 19, 369-380, 1993.

Bass, J. D., T. J. Ahrens, J. R. Abelson, and T. HuA, Shock temperatures in metals: a new result for an Fe alloy, J. Geophys. Res., 95, 21767-21776, 1990. 
BIRCH, F., The melting relations of iron, and temperatures in the earth's core, Geophys. J. R. Astron. Soc., 29, 373-387, 1972.

Boenler, R., The phase diagram of iron to 430 kbar, Geophys. Res. Lett., 13, 1153-1156, 1986.

BOEHLER, R., Melting of the Fe-O-S system at high pressures: constraints for core temperatures, Earth and Planet. Sci. Lett., 111, 217-227, 1992.

BoEhler, R., Temperatures in the Earth's core based on new static melting measurements of iron to 2 Mbar, Nature, 1993 (submitted).

BOEHLER, R., and J. RAMAKRISHNAN, Experimental results of the pressure dependence of the Grüneisen parameter: a review, J. Geophys. Res., 85, 6996-7002, 1980.

Boehler, R., N. VON BArgen, and A. Chopelas, Melting, thermal expansion and phase transitions of iron at high pressures, J. Geophys. Res., 95, 21731-21736, 1990.

Boschi, E., The melting relations of iron and temperatures in the earth's core, Riv. Nuovo Cim., 5, 501-531, 1975.

Boschi, E., F. Mulargia, and M. Bonafede, The dependence of the melting temperature on the choice of the interatomic potential, Geophys. J. R. Astron. Soc., 58, 201-208, 1979.

Brown, J. M. and R. G. MCQUeEN, The equation of state for iron in the earth's core, in High Pressure Research in Geophysics, edited by A. Akimoto and M. Manghnani, pp. 611-625, Center for Academic Publications, Tokyo, 1982.

Brown, J. M. and R. G. MCQueEn, Phase transition, Grüneisen parameter and elasticity for shocked iron between $77 \mathrm{GPa}$ and $400 \mathrm{GPa}, J$. Geophys. Res., 91, 7485-7494, 1986.

Bundy, F. P., Pressure-temperature phase diagram of iron to 200 kbar and $900^{\circ} \mathrm{C}, J$. Appl. Phys., 36, 616-620, 1965.

Bundy, F. P. and H. M. Strong, Behavior of metals at high temperature and pressure, in Solid State Physics, Vol. 13, edited by F: Seitz and D. Turnbull, pp. 81-143, Academic Press, New York, 1962.

DuBA, A., Earth's core not so hot, Nature, 359, 197-198, 1992.

Gilvarry, J. J., Temperatures of the earth's interior, J. Atmos. Terr. Phys., 10, 84-95, 1957.

Higgens, G. and G. C. Kennedy, The adiabatic gradient and the melting point gradient in the earth's core, $J$. Geophys. Res., 76, 1870-1878, 1971.

Jamieson, J. C., H. H. Demarest, and O. Schiferl, A reevaluation of the Grüneisen parameter in the earth's core, J. Geophys. Res., 83, 5929-5935, 1978.

JephCOAT, A. P. and P. OlSEn, Is the inner core of the earth pure iron?, Nature, 325, 332-335, 1987.

Kraut, E. A. and G. C. Kennedy, New melting law at high pressure, Phys. Rev., 151, 668-775, 1966.

Leppaluoto, D. A., Melting of iron by significant structure theory, Phys. Earth Planet. Inter., 6, 175-181, 1972.

LIU, L.-G., On the $(\alpha-\gamma-\ell)$ triple point of iron and the earth's core, Geophys. J. R. Astron. Soc., 43, 697-705, 1975.

LiU, L.-G. and W. A. BAssett, The melting of iron up to 200 kilobars, J. Geophys. Res., 80, 3777-3782, 1975.

MAO, H. K., W. A. BASSETT, and T. TAKAHASHI, Effect of crystal structure and lattice parameters of iron up to 300 kbar, J. Appl. Phys., 38, 272-276, 1967.

Matsui, M., Computer simulation of the structural and physical properties of iron under ultra high pressures and high temperatures, in Central Core of the Earth, Vol. 2, pp. 79-82, 1992.

MCQUEEN, R. G., Laboratory techniques for very high pressures and the behavior of metals under dynamic loading, in Conference on Metallurgy at High Pressure, edited by K. A. Gshneider, M. T. Hepworth, and N. A. D. Parlee, pp. 48-132, Gordon and Breach, New York, 1964.

MCQueEn, R. G. and D. G. IsAAK, Characterizing windows for shock wave radiation studies, J. Geophys. Res., 95, 21753-21756, 1990.

PoIRIER, J. P., Dislocation mediated melting of iron and temperature of the earth's core, Geophys. J. R. Astron. Soc., 85, 315-328, 1986.

PoIRIER, J. P. and T. Shankland, Dislocation theory of melting for iron, revisited, J. Geomag. Geoelectr., 1993 (in press).

Ringwood, A. E. and W. HibBerson, The system Fe-FeO revisited, Phys. Chem. Miner., 17, 313-319, 1990.

Ross, M., D. Young, and R. Grover, Theory of the iron phase diagram at earth-core conditions, J. Geophys. Res., 95, 21713-21715, 1990.

Shen, G., P. Lazor, and S. K. Saxena, Melting of wüstite and iron to 600 kbar, Phys. Chem. Miner., 1993 (in press).

SPILIOPOULOS, S. and F. D. STACEY, The earth's thermal profile: is there a mid-mantle thermal boundary layer?, J. Geodyn., 1, 61-77, 1984.

Stacey, F. D., A thermal model of the earth, Phys. Earth Planet. Inter., 15, 341-348, 1977.

StACEY, F. D. and R. D. IRvine, Theory of melting: thermodynamic basis of Lindemann's law, Aust. J. Phys., 30, 631-640, 1977.

Stevenson, D. J., Models of the earth's core, Science, 214, 611-618, 1981.

Strong, H. M., R. E. Tuft, and R. E. Hannemann, The iron fusion curve and the $\gamma$ - $\delta$-liquid triple point, Metall. Trans., 4, 2657-2661, 1973.

Tan, H. and T. J. Ahrens, Shock temperature measurements for metals, High Press. Res., 2, 159-182, 1990. 
Williams, Q., R. Jeanloz, J. Bass, B. Svendson, and T. J. Ahrens, Melting curve of iron to 250 GPa: A constraint on the temperature of the earth's center, Science, 236, 181-183, 1987.

Williams, Q., E. KNitTle, and R. Jeanloz, The high pressure melting curve of iron-a technical discussion, J. Geophys. Res., 96, 2171-2184, 1991.

Yoo, C. S., J. AKella, and C. M. Ruddle, Melting studies of iron and uranium by DAC laser heating, (abstract), EOS, Trans. Am. Geophys. Union, 73, 64, 1992.

Yoo, C. S., N. C. Holmes, M. Ross, D. J. WebB, and C. Pike, Shock temperatures, melting and phase diagram of iron at earth core conditions, submitted to Phys. Rev. Lett., 1993.

Young, D. A., Phase Diagrams of the Elements, University of California Press, Los Angeles, 1991.

Young, D. A. and R. Grover, Theory of the iron equation of state and melting curve to very high pressure, in Shock Waves in Condensed Matter-1983, edited by J. R. Asay, R. A. Graham, and G. K. Straub, pp. 66-67, Elsevier, New York, 1984.

Zharkov, V. N., Physics of the Earth's core, trans. (Trudy), Inst. Earth Phys. Acad. Sci., USSR, 20, $187,1962$. 